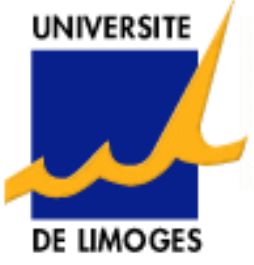

\author{
XLIM \\ UMR CNRS 6172 \\ Département \\ Mathématiques-Informatique
}

\title{
On the Moreau-Rockafellar-Robinson Qualification Condition in Banach Spaces
}

\section{Emil Ernst \& Michel Théra}

Rapport de recherche $\mathrm{n}^{\circ} 2006-02$

Déposé le 4 janvier 2006 
Université de Limoges, 123 avenue Albert Thomas, 87060 Limoges Cedex

Tél. (33) 555457323 - Fax. (33) 555457322

http://www.xlim.fr

http://www.unilim.fr/laco 


\title{
ON THE NECESSITY OF THE \\ MOREAU-ROCKAFELLAR-ROBINSON QUALIFICATION CONDITION IN BANACH SPACES
}

\author{
EMIL ERNST AND MICHEL THÉRA
}

Dedicated to Stephen Robinson in honor of his 65 th birthday.

\begin{abstract}
As well known, the Moreau-Rockafellar-Robinson internal point qualification condition is sufficient to ensure that the infimal convolution of the conjugates of two extended-real-valued convex lower semi-continuous functions defined on a locally convex space is exact, and that the sub-differential of the sum of these functions is the sum of their sub-differentials. This note is devoted to proving that this condition is, in a certain sense, also necessary, provided the underlying space is a Banach space. Our result is based upon the existence of a non-supporting weak $^{\star}$-closed hyperplane to any weak ${ }^{\star}$ - closed and convex unbounded linearly bounded subset of the topological dual of a Banach space.
\end{abstract}

\section{INTRODUCTION AND NOTATION}

Throughout $X$ will denote a real locally convex space, $X^{\star}$ its topological dual, and $\langle\cdot, \cdot\rangle$ the duality product between $X$ and $X^{\star}$. If $X$ is a Banach space, we write $\|\cdot\|$ and respectively $\|\cdot\|_{\star}$ for the norm on $X$ and the dual norm on $X^{\star}$, and $\mathbb{B}_{X^{\star}}$ is the closed unit ball in $X^{\star}$. For any extended-realvalued function $\Phi: X \rightarrow \mathbb{R} \cup\{+\infty\}$, we note Dom $\Phi$ the effective domain of $\Phi$, i.e., $\operatorname{Dom} \Phi=\{x \in X: \Phi(x)<+\infty\}$.

A central notion in the theory of convex functions is the (Fenchel-Legendre) conjugate. Let $\Phi: X \rightarrow \mathbb{R} \cup\{+\infty\}$ be a proper (not identically equal to $+\infty$, or, equivalently, Dom $\Phi \neq \emptyset$ ) extended-real-valued lower semicontinuous convex function (this class of functions is from now on denoted by $\left.\Gamma_{0}(X)\right)$. Then, the conjugate of $\Phi$ is the function $\Phi^{\star}: X^{\star} \rightarrow \mathbb{R} \cup\{+\infty\}$ defined by

$$
\Phi^{\star}\left(x^{\star}\right)=\sup _{x \in \operatorname{Dom} \Phi}\left(\left\langle x, x^{\star}\right\rangle-\Phi(x)\right) .
$$

Date: revised on January 03, 2006.

2000 Mathematics Subject Classification. 90C46, 90C51, 46N10, 49K40.

Key words and phrases. interior point constraint qualification, convex programming, Banach space, Fenchel-Legendre duality. 
This transformation has especially turned out to be essential in developing duality theory for convex optimization. Central to this purpose are the calculus rules that have been developed for expressing the conjugate of the sum of two functions in terms of their conjugates.

To this respect, let us define the concept of infimal convolution. If $\Phi$ and $\Psi$ belongs to $\Gamma_{0}(X)$, the infimal convolution of $\Phi$ and $\Psi$ is the extended real-valued function $\Phi \square \Psi$ defined by

$$
\Phi \square \Psi(x)=\inf _{y \in X}(\Phi(x-y)+\Psi(y)) .
$$

The infimal convolution between $\Phi$ and $\Psi$ is said to be exact if $\Phi \square \Psi \in \Gamma_{0}(X)$, and the infimum in relation (1) is a minimum.

Arguing that in the class $\Gamma_{0}(X)$ a function coincides with its bi-conjugate, it is a standard matter (see [15, Chap $6 \S 6.8]$ ) to prove that

$$
\Phi+\Psi=\Phi^{\star \star}+\Psi^{\star \star}=\left(\Phi^{\star} \square \Psi^{\star}\right)^{\star} .
$$

Then clearly, if Dom $\Phi \cap \operatorname{Dom} \Psi \neq \emptyset$, and if the infimal convolution between the conjugates $\Phi^{\star}$ and $\Psi^{\star}$ is exact, or equivalently, if $\operatorname{Dom} \Phi \cap \operatorname{Dom} \Psi \neq \emptyset$, and if the Minkowski sum of the epigraphs of $\Phi^{\star}$ and $\Psi^{\star}$ is weak ${ }^{\star}$-closed, then relation (2) yields that

$$
(\Phi+\Psi)^{\star}=\Phi^{\star} \square \Psi^{\star},
$$

that is the desired formula for the conjugate of the sum.

Among the many uses of relation (3), let us cite the subdifferential sum formula. Given $\Phi \in \Gamma_{0}(X)$, the operator $\partial \Phi: X \rightrightarrows X^{\star}$, defined as

$$
\partial \Phi\left(x_{0}\right)=\left\{x^{\star} \in X^{*}:\left\langle x-x_{0}, x^{\star}\right\rangle+\Phi\left(x_{0}\right) \leq \Phi(x) \quad \forall x \in X\right\},
$$

is called the subdifferential of $\Phi$. The (possibly empty) subdifferential of $\Phi$ at $x_{0}$ is related to the conjugate of $\Phi$ through the formula

$$
\Phi^{\star}\left(x^{\star}\right)+\Phi(x)=\left\langle x^{\star}, x\right\rangle \Longleftrightarrow x^{\star} \in \partial \Phi(x) .
$$

In [16], Moreau proved that, in every locally convex space $X$, the sum rule formula

$$
\partial(\Phi+\Psi)(x)=\partial \Phi(x)+\partial \Psi(x) \quad \forall x \in X,
$$

holds for every $\Phi$ and $\Psi$ in $\Gamma_{0}(X)$ provided that $\operatorname{Dom} \Phi \cap \operatorname{Dom} \Psi \neq \emptyset$, and that $\Phi$ and $\Psi$ satisfy relation (3).

Recently (see [8, Corollary 3.1]), Burachik and Jeyakumar proved when $X$ is a Banach space, that two functions $\Phi$ and $\Psi$ belonging to $\Gamma_{0}(X)$ such that $\operatorname{Dom} \Phi \cap \operatorname{Dom} \Psi \neq \emptyset$ satisfy (3), the formula for the conjugate of the sum, if and only if the Minkowski sum of the epigraphs of $\Phi^{\star}$ and $\Psi^{\star}$ is weak ${ }^{\star}$-closed, that is if and only if the subdifferential sum formula (4) holds for $\Phi$ and $\Psi$. 
Accordingly, at least for pairs of functions with non-disjoint effective domains defined on a Banach space, the validity of the formula for the conjugate of the sum, the fact that the sum of the epigraphs of the conjugates is a proper weak ${ }^{\star}$-closed set, and the sum rule formula for the subdifferential are equivalent properties.

It is thus easy to construct counter-examples for instance to the sum rule formula for the subdifferential of two $\Gamma_{0}(X)$ function, even when the underlying space $X$ is Euclidean. One manner to deal with this situation is to require a qualification condition, that is a simple geometric or analytic condition on functions $\Phi$ and $\Psi$ which suffices to ensure that relations (3-4) hold.

Perhaps the most popular among this type of conditions is the MoreauRockafellar-Robinson condition ([15, Chap $7 \oint 7.7$ Proposition 7.4]) that assumes, in the general setting of real locally convex spaces, that one of the functions is finite and continuous at some point where the other is finite.

This qualification condition rapidly knew numerous refinements, attempting in general to replace the internal point condition requiring the continuity of one of the functions, by a geometric condition concerning the interplay between their effective domains (a so-called dom-dom condition. Without the slightest claim of being exhaustive (we refer the reader to [27] for a survey on the topic), let us mention the works of Rockafellar ([22]), in reflexive Banach spaces, and Robinson ([21]), in a general Banach space, who proved that the infimal convolution of the conjugates is exact whenever $0 \in \operatorname{core}(\operatorname{Dom} \Phi-\operatorname{Dom} \Psi)$, or equivalently if the cone $\mathbb{R}_{+}(\operatorname{Dom} \Phi-\operatorname{Dom} \Psi)$ is the whole underlying space $X$. A different proof together with a slight restating of the dom-dom qualification condition was provided when Attouch and Brézis ([2]) proved, in the general setting of Banach spaces, that a sufficient condition for the exactness of the infimal convolution of the conjugates is that the convex cone spanned by the difference of the effective domains is a closed linear space.

This line of reasoning was adapted for normed spaces by Azé, (see [5]), and by Combari, Laghdir and Thibault ([10]) on one hand, and by Moussaoui and Volle (see [17] and [18]) on the other, to general locally convex spaces.

Lescarret, [14], adopted an entirely different condition, proving that relation (3), and thus also (4), holds whenever $\Phi^{\star}$ and $\Psi^{\star}$ are both minorized on $X^{\star}$ by the same sharp function with weak complete epigraph. The main result from [23, Theorem 3.2] may be used to prove that Lescarret's qualification condition implies that $\Phi$ and $\Psi$ are both finite on some set whose affine hull coincides with $X$, for instance on some Hammel basis of $X$. 
Attouch, Riahi and Théra proved ([3]) that an Attouch-Brézis type condition may equally be used to ensure that the sum of two maximal monotone operators on reflexive Banach spaces is a remains a maximal monotone operator (see also [4]), a more general setting in which the sum rule for the subdifferential obviously frames as a particular case.

The reader interested in dom-dom conditions is refereed for further reading to the excellent articles of Seetharama Gowda and Teboulle [24] and respectively Simons [25], which provide a clear picture of the topic, as well as self-contained proofs for most of the concerned results. Let us also remark the work by Verona and Verona [26] in which is given an elegant and short proof of the Attouch-Brézis result.

Although lying outside the scope of this article, let us briefly mention a different manner to face the fact that formula 4 is not always valid. Namely let us consider, instead of the convex subdifferential, the notion of $\varepsilon$-sub differential used by Hiriart-Urruty and Phelps [13] (see also [12]) to makes the sum rule for the $\varepsilon$-subdifferential valid regardless of the closedness of the Minkovski sum of the epigraphs of the functions. The same type of construction was applied to monotone operators by Revalski and Théra [20], and Fitzpatrick and Simons [11].

It is however clear that a pair of functions in $\Gamma_{0}(X)$ may satisfy relation (3), and thus the epigraph condition stating that the sum of their epigraphs is proper and weak ${ }^{\star}$-closed, even if internal point or dom-dom qualification conditions fail. For instance, when the two functions coincide, $\Phi=\Psi$, relations (3) and (4) are valid without any further condition.

Our aim is to tackle the question of the qualification condition from a different point of view. Namely, in a general Banach space, our objective is to characterize those functions $\Phi$ in $\Gamma_{0}(X)$ that are finite at some point $\bar{x} \in X$ and such that formula (3) holds for every other $\Psi \in \Gamma_{0}(X)$ which is also finite at $\bar{x} \in X$.

This task is achieved in the main result of Section 3 (Theorem 2). This result states that formula (3) holds for every $\Psi \in \Gamma_{0}(X)$ finite at $\bar{x}$ if and only if $\Phi$ is continuous at $\bar{x}$.

We may thus conclude that, in an general Banach setting, the internal point Moreau-Rockafellar-Robinson condition is not only sufficient, but, in the above mentioned sense, also necessary in order to ensure the exactness of the infimal convolution of the conjugates.

We are thus brought to disagree with the general feeling about the interplay between inner point and epigraph conditions, as stated in a very recent article [9, Abstract]: "the [epigraph] conditions are [...] much more general than the popularly known interior-point type conditions". Indeed, MoreauRockafellar-Robinson is the most comprehensive condition which, when 
applied to one of the functions $\Phi$ or $\Psi$, makes relations (3) and (4) valid, regardless of the properties of the other function.

Theorem 2 relies on the existence (established in Theorem 1, Section 2) of a non-supporting $w^{-k^{\star}}{ }^{\star}$-closed hyperplane to any weak ${ }^{\star}$-closed and convex bounded linearly bounded subset of the dual of a Banach space.

The existence of a weak ${ }^{\star}$-closed non-supporting hyperplane is no longer achieved when the underlying space is not a Banach space (see Example 1). It is an interesting question to know whether the Moreau-RockafellarRobinson qualification criterion remains necessary for the exactness of the infimal convolution of the conjugates in a general locally convex space.

\section{NON-SUPPORTING LINEAR FUNCTIONALS IN DUAL BANACH SPACES}

Let $C$ be a closed and convex subset of a topological vector space $X$. A linear and continuous map $\phi: X \rightarrow \mathbb{R}$ is non-supporting for $C$ provided that it is bounded above and does not achieve its supremum on $C$ :

$$
\phi(y)<\sup _{x \in C} \phi(x) \quad \forall y \in C .
$$

When $C$ is a closed and convex unbounded set but does not contain any half-line (such set is called linearly bounded), and $X$ is a reflexive Banach space, the existence of at least one non-supporting linear and continuous functional was stated in [1, Theorem 3.1]. Moreover, the set of all the nonsupporting linear and continuous maps is proved in [6, Theorem 1.2] to be a dense $G_{\delta}$ subset of the topological dual $X^{\star}$ of $X$.

Following some of the ideas developed in the second section of [6], we address here the question of the existence of non-supporting functionals for unbounded linearly bounded weak* closed convex subsets of the topological dual of a Banach space.

Firstly, let us state a standard property of supporting functionals of unbounded linearly bounded sets, valid for locally convex spaces.

A brief review of basic notions will help to fix notation and terminology. Given a closed convex set $C$ of the topological dual $X^{\star}$ of a locally convex space $X$, we denote by $\sigma_{C}: X \rightarrow \mathbb{R} \cup\{+\infty\}$ the support functional of $C$ defined by

$$
\sigma_{C}(x)=\sup _{y \in C}\langle x, y\rangle \text {. }
$$

For use in the sequel (Theorem 1), we need to state a technical result.

Lemma 1. Suppose that $C$ is an unbounded linearly bounded weak ${ }^{\star}$-closed and convex subset of the topological dual $X^{\star}$ of a locally convex vector space $X$. Then the closure of the level set

$$
\left[\sigma_{C}=r\right]=\left\{x \in X: \sigma_{C}(x)=r\right\}
$$


coincides with the sub-level set

$$
\left[\sigma_{C} \leq r\right]=\left\{x \in X: \sigma_{C}(x) \leq r\right\} .
$$

Let us remark that all the level sets of the support functional of a bounded weak $^{\star}$-closed and convex set are closed. Lemma 1 highlights, in the linearly bounded setting, the gap between the properties of unbounded and the properties of bounded sets.

Proof of Lemma 1: As $C$ contains no half-lines, it is well known (see [7, Chap. IV, $\S 1$, No. $3 ; 12$, page 68]) that the effective domain of $\sigma_{C}$ (that is the barrier cone of $C$ ) is weakly dense in $X$. Moreover, in any locally convex space, the weak closure and the closure of a convex set coincides (see [7, Chap. IV, $\S 2$, No. 3]), and thus Dom $\sigma_{C}$ is not only weakly dense, but also dense in $X$.

Let us prove that every super-level set

$$
\left[\sigma_{C}>r\right]=\left\{x \in X: \sigma_{C}>r\right\}
$$

is dense in $X$. Indeed, suppose, to the purpose of obtaining a contradiction, that the closure of some super-level set, say $\left[\sigma_{C}>\bar{r}\right]$ is a proper subset of $X$. As the effective domain of $\sigma_{C}$ is dense, and as

$$
\text { Dom } \sigma_{C}=\left[\sigma_{C} \leq \bar{r}\right] \cup\left[\sigma_{C}>\bar{r}\right],
$$

it follows that the difference between the underlying space $X$ and the closure of the super-level $\left[\sigma_{C}>\bar{r}\right]$ lays within the closure of the sub-level set $\left[\sigma_{C} \leq \bar{r}\right]$. Obviously,

$$
\left[\sigma_{C} \leq \bar{r}\right]=\bigcap_{y \in C}(\{x \in X:\langle x, y\rangle \leq r\}) ;
$$

being the intersection of a family of closed half-spaces, the sub-level set $\left[\sigma_{C} \leq \bar{r}\right]$ is weakly closed, thus closed, and thus coincides with its closure. Accordingly, the difference between $X$ and the closure of the super-level $\left[\sigma_{C}>\bar{r}\right]$, which is an open set, is a part of the sub-level set $\left[\sigma_{C} \leq \bar{r}\right]$.

Thus, the interior of $\left[\sigma_{C} \leq \bar{r}\right]$ is non-empty; the same obviously holds for the effective domain of $\sigma_{C}$, as it contains all the sub-level sets of $\sigma_{C}$, hence also $\left[\sigma_{C} \leq \bar{r}\right]$. Recall that in every locally convex space the closure of the interior of a convex set coincides with the closure of the convex set itself, provided that the interior is non-empty, and deduce that the interior of Dom $\sigma_{C}$ is dense in $X$. Hence Dom $\sigma_{C}=X$, and the Banach-Steinhaus Theorem states that $C$ is bounded, a contradiction.

We have thus proved that every super-level set $\left[\sigma_{C}>r\right]$ is dense in $X$. Consequently, every $x_{0} \in X$ such that $\sigma_{C}\left(x_{0}\right)<r$ is the limit of some net $\left(x_{i}\right)_{i \in I} \subset\left[\sigma_{C}>r\right]$. Restricted to any line segment of form $\left[x_{0}, x_{i}\right]$, the function $\sigma_{C}$ is convex and finite, thus continuous. We deduce that, on every 
line segment $\left[x_{0}, x_{i}\right]$ there is an element, say $y_{i}$, such that $\sigma_{C}\left(y_{i}\right)=r$. As, on one hand, the net $(y)_{i \in I} \subset\left[\sigma_{C}=r\right]$ obviously converges to $x_{0}$, and on the other, the sub-level set $\left[\sigma_{C} \leq r\right]$ is closed, we deduce that the closure of the level set $\left[\sigma_{C}=r\right]$ coincides with the sub-level set $\left[\sigma_{C} \leq r\right]$.

Our main result can also be viewed as a complement to a result of Phelps (see [19]) on supporting points in dual Banach spaces.

Theorem 1. Every weak ${ }^{\star}$-closed and convex unbounded linearly bounded subset of $X^{\star}$, the topological dual of a Banach space $X$, admits at least one non-supporting linear map of form $\langle f, \cdot\rangle, f \in X$ :

$$
\sup _{y \in C}\langle f, y\rangle=1,\langle f, x\rangle<1 \quad \forall x \in C .
$$

Proof of Theorem 1: Let $C^{\circ}=\left[\sigma_{C} \leq 1\right]$ be the polar set of $C$, and set

$$
\left(C^{\circ}\right)_{n}=\left\{x \in C^{\circ}: \sup _{y \in C,\|y\|_{\star} \leq n}\langle x, y\rangle=\sup _{y \in C}\langle x, y\rangle\right\}
$$

for the set of elements $x$ from $C^{\circ}$ such that the linear map $\langle x, \cdot\rangle$ achieves its supremum on $C$ within the ball of radius $n$ of $X^{*}$.

It is a standard matter to prove that every $\left(C^{\circ}\right)_{n}$ is weakly closed. Indeed, let $x_{0}$ be a the weak limit of the net $\left(x_{i}\right)_{i \in I} \subset\left(C^{\circ}\right)_{n}$. Obviously,

$$
\sup _{y \in A}\left\langle x_{0}, y\right\rangle \leq \liminf _{i \in I}\left(\sup _{y \in A}\left\langle x_{i}, y\right\rangle\right)
$$

for every subset $A$ of $X^{\star}$, but

$$
\sup _{y \in B}\left\langle x_{0}, y\right\rangle=\lim _{i \in I}\left(\sup _{y \in B}\left\langle x_{i}, y\right\rangle\right)
$$

when the subset $B$ of $X^{\star}$ is weak ${ }^{\star}$-compact.

Apply relations (7) for $A=C$ and (8) for $B=\left\{y \in C:\|y\|_{\star} \leq n\right\}$ (a weak ${ }^{\star}$-compact set by virtue of the Alaoglu-Bourbaki Theorem) and use relation

$$
\sup _{y \in C}\left\langle x_{i}, y\right\rangle=\sup _{y \in C,\|y\|_{\star} \leq n}\left\langle x_{i}, y\right\rangle
$$

(in other words the fact that $x_{i} \in\left(C^{\circ}\right)_{n}$ ) to deduce that

$$
\begin{aligned}
\sup _{y \in C}\left\langle x_{0}, y\right\rangle & \geq \sup _{y \in C,\|y\|_{*} \leq n}\left\langle x_{0}, y\right\rangle=\lim _{i \in I}\left(\sup _{y \in C,\|y\|_{*} \leq n}\left\langle x_{i}, y\right\rangle\right) \\
& =\lim _{i \in I}\left(\sup _{y \in C}\left\langle x_{i}, y\right\rangle\right) \geq \sup _{y \in C}\left\langle x_{0}, y\right\rangle .
\end{aligned}
$$

Accordingly, all the terms in relation (9) coincide. Thus, on one hand, $x_{0}$ satisfies relation

$$
\sup _{y \in C}\left\langle x_{0}, y\right\rangle=\sup _{y \in C,\|y\|_{\star} \leq n}\left\langle x_{0}, y\right\rangle
$$


and on the other $x_{0} \in C^{\circ}$, as the weak limit of a net itself laying within the weakly closed (see relation 5) set $C^{\circ}$. Accordingly, all the limit points of converging nets from $\left(C^{\circ}\right)_{n}$ belong to $\left(C^{\circ}\right)_{n}$, which turns thus to be a weakly closed set.

The set $C^{\circ}$ is a closed subset of the complete metric space $X$, while $\left(C^{\circ}\right)_{n}$ are closed subsets of $C^{\circ}$. Let us suppose, to the end of achieving a contradiction, that the relative interior with respect to $C^{\circ}$ of the closure of the union of sets $\left(C^{\circ}\right)_{n}$ is non-empty. The Baire Category Theorem implies that, in this case, the interior relative to $C^{\circ}$ of at least one of the sets $\left(C^{\circ}\right)_{n}$, say $\left(C^{\circ}\right)_{\bar{n}}$, is non-empty.

Accordingly, there is $\bar{x} \in\left(C^{\circ}\right)_{\bar{n}}$ and $\varepsilon>0$ such that

$$
\left(\bar{x}+\varepsilon \mathbb{B}_{X^{*}}\right) \cap C^{\circ} \subset\left(C^{\circ}\right)_{\bar{n}} .
$$

Set

$$
\bar{y}=\left\{\begin{array}{cl}
\bar{x} & \text { if } \sigma_{C}(\bar{x})<1 \\
\frac{\bar{x}}{2} & \text { if } \sigma_{C}(\bar{x})=1 \text { and }\|\bar{x}\| \leq \varepsilon \\
\left(1-\frac{\varepsilon}{2\|\bar{x}\|}\right) \bar{x} & \text { if } \sigma_{C}(\bar{x})=1 \text { and }\|\bar{x}\|>\varepsilon
\end{array} .\right.
$$

Relation (10) implies, on one hand, that $\|\bar{x}-\bar{y}\| \leq \frac{\varepsilon}{2}$, which means that

$$
\left(\bar{y}+\frac{\varepsilon}{2} \mathbb{B}_{X^{*}}\right) \cap C^{\circ} \subset\left(\bar{x}+\varepsilon \mathbb{B}_{X^{*}}\right) \cap C^{\circ} \subset\left(C^{\circ}\right)_{\bar{n}},
$$

and on the other that

$$
\sigma_{C}(\bar{y})<1 \text {. }
$$

As $\bar{y} \in\left[\sigma_{C} \leq 1\right]$, use Lemma 1 to deduce that $\bar{y}$ is the limit of a sequence $\left(y_{m}\right)_{m \in \mathbb{N}^{*}} \subset\left[\sigma_{C}=1\right]$

Apply relation (8) for $B=\left\{z \in C:\|z\|_{\star} \leq \bar{n}\right\}$ to deduce that

$$
\sup _{y \in C,\|z\|_{\star} \leq \bar{n}}\langle\bar{y}, z\rangle=\lim _{m \rightarrow \infty}\left(\sup _{y \in C,\|z\|_{\star} \leq \bar{n}}\left\langle y_{m}, z\right\rangle\right),
$$

and as $\sup _{y \in C,\|z\|_{\star} \leq \bar{n}}\langle\bar{y}, z\rangle \leq \sigma_{C}(\bar{y})$, use relation (12) to infer that, for $m$ large enough,

$$
\sup _{y \in C,\|z\|_{\star} \leq \bar{n}}\left\langle y_{m}, z\right\rangle<1
$$

As $\sup _{y \in C}\left\langle y_{m}, z\right\rangle=1$ for every $m \in \mathbb{N}^{*}$, it follows that, at least for large enough $m, y_{n} \notin\left(C^{\circ}\right)_{\bar{n}}$.

Consequently, $\bar{y}$ is the limit of a sequence laying within $C^{\circ}$ but without $\left(C^{\circ}\right)_{\bar{n}}$. This fact visibly contradicts relation (11). 
We have therefore proved that the interior relative to $C^{\circ}$ of the closure of the union of sets $\left(C^{\circ}\right)_{n}$ is empty. In other words, it follows that the set $C^{\circ} \backslash\left(\bigcup_{n \in \mathbb{N}^{*}}\left(C^{\circ}\right)_{n}\right)$ is dense in $C^{\circ}$.

The level set $\left[\sigma_{C}=1\right]$ is a non-empty (its closure is the sub-level set $\left.\left[\sigma_{C} \leq 1\right]=C^{\circ}\right)$ part of $C^{\circ}$; we may therefore pick $z_{0} \in\left[\sigma_{C}=1\right]$ and $\left(z_{m}\right)_{m \in \mathbb{N}^{*}} \subset\left(C^{\circ} \backslash\left(\bigcup_{n \in \mathbb{N}^{*}}\left(C^{\circ}\right)_{n}\right)\right)$ such that the sequence $z_{m}$ converges to $z_{0}$.

The sub-level sets of the support function are closed (in conformity with relation 5); the function $\sigma_{C}$ is thus lower-semi-continuous. Accordingly, $\sigma_{C}\left(z_{0}\right) \leq \liminf _{m \rightarrow \infty} \sigma_{C}\left(z_{m}\right)$, and, as $\sigma_{C}\left(z_{0}\right)=1$, it follows that, for $m$ large enough, say $m \geq m_{0}$, it holds $\sigma_{C}\left(z_{m}\right)>\frac{1}{2}$. Pick $k>m_{0}$ and set $f=$ $\frac{z_{k}}{\sigma_{C}\left(z_{k}\right)}$. Obviously $\sup _{y \in C}\langle f, y\rangle=1$, while, as $\sup _{y \in C,\|y\|_{\star} \leq n}\left\langle z_{k}, y\right\rangle<$ $\sup _{y \in C}\left\langle z_{k}, y\right\rangle$ for every $n \in \mathbb{N}^{*}$,

$$
\begin{aligned}
\langle f, x\rangle & =\frac{1}{\sigma_{C}\left(z_{k}\right)}\left\langle z_{k}, x\right\rangle \leq \frac{1}{\sigma_{C}\left(z_{k}\right)} \sup _{y \in C,\|y\|_{\star} \leq n}\left\langle z_{k}, y\right\rangle \\
& <\frac{1}{\sigma_{C}\left(z_{k}\right)} \sup _{y \in C}\left\langle z_{k}, y\right\rangle=1
\end{aligned}
$$

for every $x$ in $C^{\circ}$ and $n \in \mathbb{N}^{*}$ such that $\|x\|_{\star} \leq n$. Relation (6) is thus fulfilled for $f=\frac{z_{k}}{\sigma_{C}\left(z_{k}\right)}$.

The next example proves that the Banach setting cannot be relaxed in Theorem 1.

Example 1. Let $H$ be a separable Hilbert space with basis $\left(b_{n}\right)_{n \in \mathbb{N}^{*}}$, and set $X$ for the vector space spanned by the set $\left\{b_{i}: i \in \mathbb{N}^{*}\right\}$; when endowed with the norm induced by the Hilbert product, $X$ is a non complete normed vector space whose topological dual can be identified with $H$.

Set $C=\left\{x \in H=X^{\star}:-1 \leq x \cdot b_{n} \leq 1 \quad \forall n \in \mathbb{N}^{*}\right\}$ for the Hilbert cube of $H$ (here $\cdot$ denotes the Hilbert space product), a weak ${ }^{\star}$-closed and convex linearly bounded set. Any $f \in X$ is a finite linear combination of elements from the basis $\left(b_{n}\right)$, that is $f=\sum_{i=1}^{i=n} f_{i} b_{i}$ for some $n \in \mathbb{N}^{*}$ and $f_{i} \in \mathbb{R}$ for $1 \leq i \leq n$. It is clear that $\sup _{x \in C}\langle f, x\rangle=\sum_{i=1}^{i=n}\left|f_{i}\right|$, and that the linear map $\langle f, \cdot\rangle$ achieves its supremum at every point of form $x=\sum_{i=1}^{i=n}(-1)^{g_{i}} b_{i}$, where $g_{i}=1$ if $f_{i} \geq 0$ and $g_{i}=-1$ if $f_{i}<0$.

Accordingly, every linear map $\langle f, \cdot\rangle, f \in X$, is supporting for the set $C$.

\section{THE MAIN RESULT}

We turn now to the main result of the paper.

Theorem 2. Let $X$ be a Banach space and $\Phi \in \Gamma_{0}(X)$ such that $\Phi(\bar{x}) \in \mathbb{R}$ for some $\bar{x} \in X$. The following statements are equivalent: 
i) $\Phi$ is continuous at $\bar{x}$;

ii) the Moreau-Rockafellar formula

$$
(\Phi+\Psi)^{*}=\Phi^{*} \square \Psi^{*}
$$

holds and the infimal convolution is exact for every $\Psi \in \Gamma_{0}(X)$ such that $\Psi(\bar{x}) \in \mathbb{R}$.

iii) the subdifferential sum formula

$$
\partial(\Phi+\Psi)=\partial \Phi+\partial \Psi
$$

holds for every $\Psi \in \Gamma_{0}(X)$ such that $\Psi(\bar{x}) \in \mathbb{R}$.

Proof of theorem 2: (ii) $\Leftrightarrow$ (iii) Apply [8, Corollary 3.1].

$(i) \Longrightarrow($ ii $)$ Apply the Moreau-Rockafellar Theorem ([15, Chap $7 \S 7.7$ Proposition 7.4]).

$($ ii $) \Longrightarrow(i)$ Without any lost of generality, we may (and will) suppose that $\bar{x}=0$. Indeed, it is easy to verify that, for every $\bar{x} \in X$, the MoreauRockafellar formula holds, and that the infimal convolution is exact for a pair of $\Gamma_{0}(X)$ functions $\Phi$ and $\Psi$, if and only if the same holds for the functions $\Phi_{\bar{x}}(x):=\Phi(x+\bar{x})$ and $\Psi_{\bar{x}}(x):=\Psi(x+\bar{x})$

It is well known (see [15, Chap $7 \S 7.1$ Proposition 7.1]) that a function in $\Gamma_{0}(X)$ is continuous at 0 if and only if all the sub-level sets of its conjugate are bounded. Accordingly, once statement $(i i)$ is assumed, we shall prove that all the sub-level sets $\left[\Phi^{\star} \leq r\right]$ of the conjugate $\Phi^{\star}$ of $\Phi$ are bounded in $X^{\star}$.

We start by establishing that if statement $(i i)$ is true, then all the sub-level sets of $\Phi^{\star}$ are linearly bounded. Suppose, on the contrary, that one of the sub-level sets of $\Phi^{\star}$ contains a half-line; in other words,

$$
\Phi^{\star}\left(x_{0}+\lambda x_{1}\right) \leq r \quad \forall \lambda \geq 0
$$

for some $x_{0}, x_{1} \in X^{\star}$. Then define the convex function $\phi:[0,+\infty[\rightarrow \mathbb{R}$, $\phi(s)=\Phi^{\star}\left(x_{0}+s x_{1}\right)$. For every convex function $\psi:[0,+\infty[\rightarrow \mathbb{R}$ such that $\psi\left(s_{1}\right)<\psi\left(s_{2}\right)$ for some $0 \leq s_{1}<s_{2}$, obviously $\lim _{s \rightarrow+\infty} \psi(s)=+\infty$. Apply the previous remark to the convex mapping $\phi$ to deduce, in accord with relation (13), that $\phi$ is decreasing on $[0,+\infty[$.

Recall that $\Phi(0)<+\infty$, which implies that $\Phi^{\star}$ is bounded below on $X^{\star}$, to deduce that $\phi$ is bounded below on $\left[0,+\infty\left[\right.\right.$, and set $d=\inf _{s \geq 0} \phi(s)$. As $\phi$ is a decreasing function bounded below by $d$ it results that

$$
\phi(t) \geq d \quad \forall t \geq 0 \text { and } \lim _{s \rightarrow \infty} \phi(s)=d .
$$

Set

$$
\Psi: X \rightarrow \mathbb{R} \cup\{+\infty\}, \Psi(x)=\left\{\begin{array}{cc}
-2 \sqrt{\left\langle x, x_{1}\right\rangle} & \text { if }\left\langle x, x_{1}\right\rangle \geq 0 \\
+\infty & \text { if }\left\langle x, x_{1}\right\rangle<0
\end{array} ;\right.
$$


obviously, $\Psi \in \Gamma_{0}(X)$ and $\Psi(0)=0<+\infty$. Moreover,

$$
\Psi^{\star}(x)=\left\{\begin{array}{cl}
\frac{1}{s} & \text { if } x=-s x_{1} \text { for some } s \geq 0 \\
+\infty & \text { elswhere. }
\end{array}\right.
$$

Let us prove that the infimal convolution $\Phi^{\star} \square \Psi^{\star}$ is not exact at $x_{0}$. Indeed, using relation (14) we deduce that

$$
\Phi^{\star}\left(x_{0}+s x_{1}\right)+\Psi^{\star}\left(-s x_{1}\right)=\phi(s)+\frac{1}{s}>d \quad \forall s \geq 0 ;
$$

We observe easily that if $x \notin\left\{x_{0}+\mathbb{R}_{+} x_{1}\right\}$, then $\left(x_{0}-x\right) \notin\left\{-\mathbb{R}_{+} x_{1}\right\}$, and thus $\Psi^{\star}\left(x_{0}-x\right)=+\infty$. Relation (15) and the previous remark yield

$$
\Phi^{\star}(x)+\Psi^{\star}\left(x_{0}-x\right)>d \quad \forall x \in X^{*} .
$$

Use once again relation (14) to deduce that

$$
\begin{aligned}
\Phi^{\star} \square \Psi^{\star}\left(x_{0}\right) & \leq \inf _{s \geq 0}\left(\Phi^{\star}\left(x_{0}+s x_{1}\right)+\Psi^{\star}\left(-s x_{1}\right)\right) \\
& =\inf _{s \geq 0}\left(\phi(s)+\frac{1}{s}\right)=d ;
\end{aligned}
$$

combining relations (16) and (17) yields

$$
\Phi^{\star}(x)+\Psi^{\star}\left(x_{0}-x\right)>\Phi^{\star} \square \Psi^{\star}\left(x_{0}\right) \quad \forall x \in X^{\star} .
$$

Accordingly, the infimal convolution $\Phi^{\star} \square \Psi^{\star}$ is not exact at $x_{0}$.

This fact contradicts statement $(i i)$, and therefore. statement $(i i)$ implies that all the sub-level sets of $\Phi^{\star}$ are linearly bounded.

Finally, let us prove that, when statement $(i i)$ holds, then the sub-level sets of $\Phi^{\star}$ are not only linearly bounded, but also norm-bounded. Indeed, suppose, on the contrary that a sub-level set of $\Phi^{\star}$, say $\left[\Phi^{\star} \leq r\right]$ is unbounded. Apply Theorem 1 to the unbounded linearly bounded weak ${ }^{\star}$ closed and convex set $\left[\Phi^{\star} \leq r\right]$ to deduce the existence of $x_{1} \in X$ such that

$$
\left\langle x_{1}, y\right\rangle<1 \quad \forall y \in\left[\Phi^{\star} \leq r\right] \text { and } \sup _{z \in\left[\Phi^{\star} \leq r\right]}\left\langle x_{1}, z\right\rangle=1
$$

Set

$$
\Psi(x)=\left\{\begin{array}{cl}
r-s & \text { if } x=s x_{1} \text { for some } s \in \mathbb{R} \\
+\infty & \text { elsewhere }
\end{array} ;\right.
$$

clearly $\Psi \in \Gamma_{0}(X)$, and fulfills $\Psi(0)=r<+\infty$, as well as

$$
\Psi^{\star}(x)= \begin{cases}-r & \text { if }\left\langle x_{1}, x\right\rangle=-1 \\ +\infty & \text { elswhere. }\end{cases}
$$


Let us prove that the infimal convolution $\Phi^{\star} \square \Psi^{\star}$ is not exact at 0 . From relation (18) it results on one hand, that $\Phi^{*}(x)>r$ provided that $\left\langle x, x_{1}\right\rangle=$ 1 ; as $\Psi^{\star}(-x)=-r$ for every $x \in X^{\star}$ such that $\left\langle x, x_{1}\right\rangle=1$, it follows that

$$
\Phi^{\star}(x)+\Psi^{\star}(-x)>0 \quad \forall x \in X,\left\langle x, x_{1}\right\rangle=1 .
$$

For every $x \in X$ such that $\left\langle x, x_{1}\right\rangle \neq 1$ we have $\Psi^{\star}(-x)=+\infty$; this reasoning, together with relation (19), implies that

$$
\Phi^{\star}(x)+\Psi^{\star}(-x)>0 \quad \forall x \in X .
$$

On the other hand, relation (18) implies that there is a sequence $\left(y_{n}\right)_{n \in \mathbb{N}^{*}}$ in $\left[\Phi^{\star} \leq r\right]$ such that $\lim _{n \rightarrow \infty}\left\langle x_{1}, y_{n}\right\rangle=1$. Pick $y_{0} \in X^{\star}$ such that $\left\langle x_{1}, y_{0}\right\rangle=1$; obviously, $\left\langle x_{1}, y_{n}+\left(1-\left\langle x_{1}, y_{n}\right\rangle\right) y_{0}\right\rangle=1$, which means that $\Psi^{\star}\left(-y_{n}-\left(1-\left\langle x_{1}, y_{n}\right\rangle\right) y_{0}\right)=-r$. Accordingly, for every $n \in \mathbb{N}^{*}$,

$$
\begin{aligned}
& \Phi^{*} \square \Psi^{\star}\left(\left(\left\langle x_{1}, y_{n}\right\rangle-1\right) y_{0}\right) \\
& \quad \leq \Phi^{\star}\left(y_{n}\right)+\Psi^{\star}\left(-y_{n}-\left(1-\left\langle x_{1}, y_{n}\right\rangle\right) y_{0}\right) \leq r-r=0 .
\end{aligned}
$$

From statement $(i i)$ it follows that the infimal convolution $\Phi^{\star} \square \Psi^{\star}$ is a lower semi-continuous function; as the sequence $\left(\left\langle x_{1}, y_{n}\right\rangle-1\right) y_{0}$ obviously converges to 0 , relation (21) implies that

$$
\Phi^{\star} \square \Psi^{\star}(0) \leq \liminf _{n \in \mathbb{N}^{*}}\left(\Phi^{\star} \square \Psi^{\star}\left(\left(\left\langle x_{1}, y_{n}\right\rangle-1\right) y_{0}\right)\right) \leq 0 .
$$

Relations (20) and (22) prove that the infimal convolution $\Phi^{\star} \square \Psi^{\star}$ is not exact at 0 , a contradiction. We have thus proved that statement ( $i i$ ) implies that any sub-level set of $\Phi^{\star}$ is bounded, that is $\Phi$ is continuous at 0 .

Example 1 proves that Theorem 1, which states the existence of at least one weak ${ }^{\star}$-closed non-supporting hyperplane for every unbounded linearly bounded weak ${ }^{\star}$-closed and convex set, is no longer verified in a non-Banach setting.

However, it is (at our knowledge) still an open question whether the Moreau-Rockafellar-Robinson qualification criterion remains necessary for the exactness of the infimal convolution of the conjugates in a general locally convex space or not.

In particular, it is not clear whether, when the underlying space is the noncomplete normed space $X$ described at Example 1, the mapping $\Phi(f)=$ $\sum_{i=1}^{i=n}\left|f_{i}\right|$ (which belongs to $\Gamma_{0}(X)$ and is not continuous at 0 ) fulfills statement $(i i)$ of Theorem 2 or not.

\section{REFERENCES}

[1] S. AdLy, E. ERnst and M. ThÉRA, A Characterization of Convex and Semicoercive Functionals, J. Convex Anal., vol. 8, nr. 1(2001), 127-148. 
[2] H. Attouch, H. BrÉzis, Duality for the sum of convex functions in general Banach spaces, Aspects of mathematics and its applications, Collect. Pap. Hon. L. Nachbin, 125-133, 1986.

[3] H. Attouch, H. Riahi, M. ThÉRA, Somme ponctuelle d'op?rateurs maximaux monotones, Serdica Math. J. 22 nr. 3(1996), 165-190.

[4] H. Attouch, M. Théra, A General Duality Principle for the Sum of Two Operators, J. Convex Anal., vol. 3 nr. 1(1996), 1-24.

[5] D. AzÉ, Duality for the sum of convex functions in general normed spaces, Arch.Math., vol. 62 (1994), 554-561.

[6] J. Borwein, I. KorTezov, Some generic results on nonattaining functionals, Setvalued Anal., vol. 9, nr. 1-2(2001), 35-47.

[7] N. Bourbaki, Elements de Mathématique, Livre V, Espaces Vectoriels Topologiques, Fasc. XVIII, Hermann, Paris, 1964.

[8] R.S. BurAChiK, V. JeYAKUmar, A dual condition for the convex subdifferential sum formula with applications, J. Convex Anal., vol. 12 nr. 2(2005), 279-290.

[9] R.S. BURACHIK, V. JEYAKUMAR and Z.-Y. WU, Necessary and sufficient conditions for stable conjugate duality, Nonlin. Anal.: T.M.A., to appear.

[10] C. Combari, M. LAghdir and L. Thibault, On Sub-differential Calculus for Convex Functions Defined on Locally Convex Spaces, Ann. Sci. Math. Québec, vol. 23 (1999), 23-36.

[11] S. P. FitzPATRICK, S. SimOnS, The conjugates, compositions and marginals of convex functions, J. Convex Anal. vol. 8 (2001) no. 2, 423-446.

[12] J.-B. Hiriart-Urruty, M. Moussaoui, A. Seeger, M. Volle, Subdifferential calculus without qualification conditions, using approximate subdifferentials: a survey, Nonlinear Anal. vol. 24 (1995), 1727-1754.

[13] J.-B. Hiriart-Urruty, R. R. Phelps, Subdifferential calculus using $\varepsilon-$ subdifferentials, J. Functional Analysis, vol. 118 (1993), 154-166.

[14] C. LESCARRET, Sur la sous-différentiabilité d'une somme de fonctionnelles convexes semi-continues inférieurement, C. R. Acad. Sci. Paris, Sér. A, vol. 262 (1966), 443446.

[15] J.J. Moreau, Fonctionnelles convexes, Seminaire sur les Équations aux dérivées partielles, Collège de France, Paris, 1967.

[16] J.J. Moreau, Etude locale d'une fonctionnelle convexe, Séminaires de Mathématique, Faculté des Sciences de Montpellier, 1963.

[17] M. Moussaoui, M. Volle, Sur la quasicontinuité et les fonctions unies en dualité convexe, C. R. Acad. Sci. Paris Sér. I, vol. 322 (1996), 839-844.

[18] M. Moussaoui, M. Volle, Quasicontinuity and united functions in convex duality theory, Comm. Appl. Nonlinear Anal, vol. 4 (1997), 73-89.

[19] R. R. PHELPS, Weak* support points of convex sets in $E^{*}$, Israel J. Math., 2 (1964), 177-182.

[20] J. REVAlski, M. THÉRA, Enlargements and sums of monotone operators, Nonlinear Anal., Ser. A: Theory Methods, vol. 48 nr. 4(2002), 505-519.

[21] S.M. Robinson, Regularity and stability for convex multivalued functions, Math. Oper. Res. 1 (1976), 130-143.

[22] R.T. RockAfEllar, Conjugate duality and optimisation, SIAM Publications, Philadelphia, 1974.

[23] J. SAINT-PIERre, M. VALADIER, Functions with sharp weak completely epigraphs, J. Convex Anal., vol. 1, nr. 1(1994), 101-105. 
[24] M. Seetharama Gowda, M. Teboulle, A comparison of constraint qualifications in infinite-dimensional convex programming, SIAM J. Control Optim., vol. 28 nr. 4(1990), 925-935.

[25] S. Simons, Sum theorems for monotone operators and convex functions, Trans. Amer. Math. Soc., vol. 350 nr. 7(1998), 2953-2972.

[26] A. Verona, M. E. Verona, A simple proof of the sum formula, Bull. Austral. Math. Soc., vol. 63 (2001), 337-339.

[27] C. ZăLINESCU, A comparison of constraint qualifications in infinite dimensional convex programming revisited, J. Aust. Math. Soc. Ser. B, 40 3(1999), 353-378.

Laboratoire de Modelis ation en Mécanique et Thermodynamique, Case 322, Université Paul Cézanne Aix-Marseille III, Avenue Escadrille NorMANDIE-NIEMEN 13397 MARSEILlE CEDEX 20

E-mail address: Emil.Ernst@univ.u-3mrs.fr

Xlim, Université de Limoges, 123 Avenue A. Thomas, 87060 Limoges CEDEX, France

E-mail address: michel.thera@unilim.fr 